\title{
KOBIO, the First Web-based Korean Biologics Registry Operated With a Unified Platform Among Distinct Disease Entities
}

Jinhyun Kim, M.D., Ph.D. ${ }^{1, *}$, Jung Hee Koh, M.D., Ph.D. ${ }^{2, *}$, Sung Jae Choi, M.D., Ph.D. ${ }^{3}$, Chan Hong Jeon, M.D., Ph.D. ${ }^{4}$, Seung-Ki Kwok, M.D., Ph.D. ${ }^{5}$, Seong-Kyu Kim, M.D., Ph.D. ${ }^{6}$, Chan-Bum Choi, M.D., Ph.D. ${ }^{7}$, Jaejoon Lee, M.D., Ph.D. ${ }^{8}$, Changhoon Lee, M.D., Ph.D. ${ }^{9}$, Eon Jeong Nam, M.D., Ph.D. ${ }^{10}$, Yong-Beom Park, M.D., Ph.D. ${ }^{11}$, Shin-Seok Lee, M.D., Ph.D. ${ }^{12}{ }^{\prime}$, Tae-Hwan Kim, M.D., Ph.D. ', Sung-Hwan Park, M.D., Ph.D. ${ }^{5}$, Jung-Yoon Choe, M.D., Ph.D. ${ }^{6}$, Eun-Mi Koh, M.D., Ph.D. ${ }^{\prime}$, Dae-Hyun Yoo, M.D., Ph.D. ${ }^{7}$, Yeong Wook Song, M.D., Ph.D. ${ }^{13}$, Hyoun-Ah Kim, M.D., Ph.D. ${ }^{14},{ }^{\prime}$ Kichul Shin, M.D., Ph.D. ${ }^{15}$

${ }^{1}$ Department of Internal Medicine, Chungnam National University College of Medicine, Daejeon, ${ }^{2}$ Division of Rheumatology, Department of Internal Medicine, Bucheon St. Mary's Hospital, College of Medicine, The Catholic University of Korea, Seoul, ${ }^{3}$ Division of Rheumatology, Department of Internal Medicine, Korea University Ansan Hospital, Ansan, ${ }^{4}$ Division of Rheumatology, Department of Internal Medicine, Soonchunhyang University Bucheon Hospital, Bucheon, ${ }^{5}$ Division of Rheumatology, Department of Internal Medicine, Seoul St. Mary's Hospital, College of Medicine, The Catholic University of Korea, Seoul, ${ }^{6}$ Division of Rheumatology, Department of Internal Medicine, Daegu Catholic University School of Medicine, Daegu, ${ }^{7}$ Department of Rheumatology, Hanyang University Hospital for Rheumatic Diseases, Seoul, ${ }^{8}$ Department of Medicine, Samsung Medical Center, Sungkyunkwan University School of Medicine, Seoul, ${ }^{9}$ Department of Internal Medicine, Wonkwang University Hospital, Iksan, ${ }^{10}$ Department of Internal Medicine, Kyungpook National University School of Medicine, Daegu, ${ }^{11}$ Division of Rheumatology, Department of Internal Medicine, Yonsei University College of Medicine, Seoul, ${ }^{12}$ Department of Rheumatology, Chonnam National University Hospital, Gwangju, ${ }^{13}$ Division of Rheumatology, Department of Internal Medicine, Seoul National University Hospital, Seoul, ${ }^{14}$ Department of Rheumatology, Ajou University School of Medicine, Suwon, ${ }^{15}$ Division of Rheumatology, Department of Internal Medicine, Seoul Metropolitan Government-Seoul National University Boramae Medical Center, Seoul, Korea

The KOrean College of Rheumatology BIOlogics and targeted therapy (KOBIO) registry is a nationwide observational cohort that captures detailed data on exposure of patients to biologic and targeted synthetic disease-modifying anti-rheumatic drugs (DMARDs). This registry was launched in December 2012 with an aim to prospectively investigate clinical manifestations and outcomes of patients with rheumatoid arthritis (RA), ankylosing spondylitis, and psoriatic arthritis who initiated a biologic or targeted synthetic DMARD or switched to another. Demographic data, disease activity, current treatment, adverse events, terms based on Medical Dictionary for Regulatory Activities, and so on are registered for patients who are then followed up annually in a web-based unified platform. The KOBIO registry also recruits and collects data of patients with RA on conventional DMARDs for comparison. As of today, more than 5,500 patients were enrolled from 47 academic and community Rheumatology centers across Korea. The KOBIO registry has evolved to become a powerful database for clinical research to improve clinical outcomes and quality of treatment. (J Rheum Dis 2021;28:176-182)

Key Words. Registries, Biological products, Rheumatoid arthritis, Ankylosing spondylitis, Psoriatic arthritis

\section{INTRODUCTION}

Real-world data (RWD) refer to observational data de- rived from diverse sources such as electronic health records, healthcare databases, claims, billing databases, disease registries, and data gathered through personal de-

\footnotetext{
Received : June 28, 2021, Revised : July 11, 2021, Accepted : July 26, 2021

Corresponding to : Kichul Shin (1D http://orcid.org/0000-0002-6749-7598

Division of Rheumatology, Department of Internal Medicine, Seoul Metropolitan Government-Seoul National University Boramae Medical Center, 20 Boramae-ro 5-gil, Dongjak-gu, Seoul 07061, Korea. E-mail : kideb1@gmail.com Hyoun-Ah Kim (iDhttp://orcid.org/0000-0003-2609-3367

Department of Rheumatology, Ajou University School of Medicine, 164 WorldCup-ro, Yeongtong-gu, Suwon 16499, Korea. E-mail : nakhada@naver.com

*These authors contributed equally to this work
}

Copyright (c) 2021 by The Korean College of Rheumatology.

This is an Open Access article, which permits unrestricted non-commerical use, distribution, and reproduction in any medium, provided the original work is properly cited. 
vices and health applications [1,2]. RWD complements the knowledge gained from clinical trials known to have limitations such as boundaries of inclusion/exclusion, low number of subjects, and short follow-up periods [3]. In contrast, a registry is a representation of collected RWD and an organized system that uses study methods to obtain observational data and evaluate specified outcomes for a population defined by a particular disease, condition, or exposure [4].

The discovery and introduction of biologics in the early 21 st century have revolutionized the treatment of rheumatic disease, particularly rheumatoid arthritis (RA). In line with that, nationwide biologics registers are established to monitor patients treated with biologics and to evaluate their long-term safety and effectiveness [5]. Thanks to biologics registers, effectiveness and safety data of biologics including latent and rare outcomes are now captured and analyzed for hundreds of thousands of patients around the world [6-8]. Direct comparative studies of different biologics can be performed based on register data $[9,10]$. Such studies are rarely performed through clinical trials. Besides, register data could provide insight of how clinical practice evolves.

The KOrean College of Rheumatology (KCR) BIOlogics and targeted therapy (KOBIO) registry was established in December 2012. The registry enrolls patients with RA, ankylosing spondylitis (AS), and psoriatic arthritis (PsA) when the patient initiates a new biologic or targeted synthetic (ts-) DMARD or switches to another. The KOBIO registry has built a robust, high-quality, and web-based platform for data collection, which is ideal for database management, access, and extraction. As for clinical safety data, the Medical Dictionary for Regulatory Activities (MedDRA), a structured terminology developed by the International Council for Harmonization of Technical Requirements for Pharmaceuticals for Human Use (ICH), was implemented for recording standardized information [11]. In this review, we describe the overall aspect of the KOBIO registry including the aim, history, organization, data platform, MedDRA, its footprints, and future directions.

\section{OVERVIEW OF KOBIO}

The KOBIO registry project was spearheaded by the Scientific Committee of the KCR (Chair Shin-Seok Lee) in late 2011 and commissioned by the then Chairman Yeong Wook Song. The aim of KOBIO was to investigate known or novel adverse events in biologics-treated patients diagnosed with rheumatic disease. Members of the Committee were divided into three groups with each group developing case report forms (CRF) for RA, AS, and PsA, respectively. Kwok et al. have extensively reviewed the schematic and governance of European RA biologic registers (BSRBR, DANBIO, etc.). Choi et al. have adapted the Charlson Comorbidity Index and Kim et al. have shared previous experiences of the Hanyang AS register. The KOBIO-RA registry initially planned to en-

\section{A}

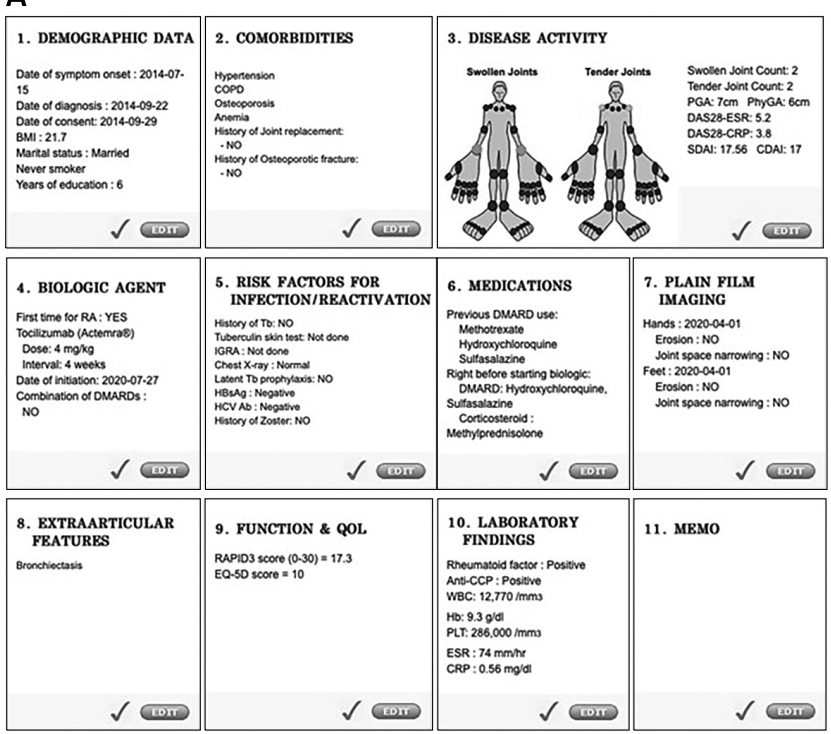

B

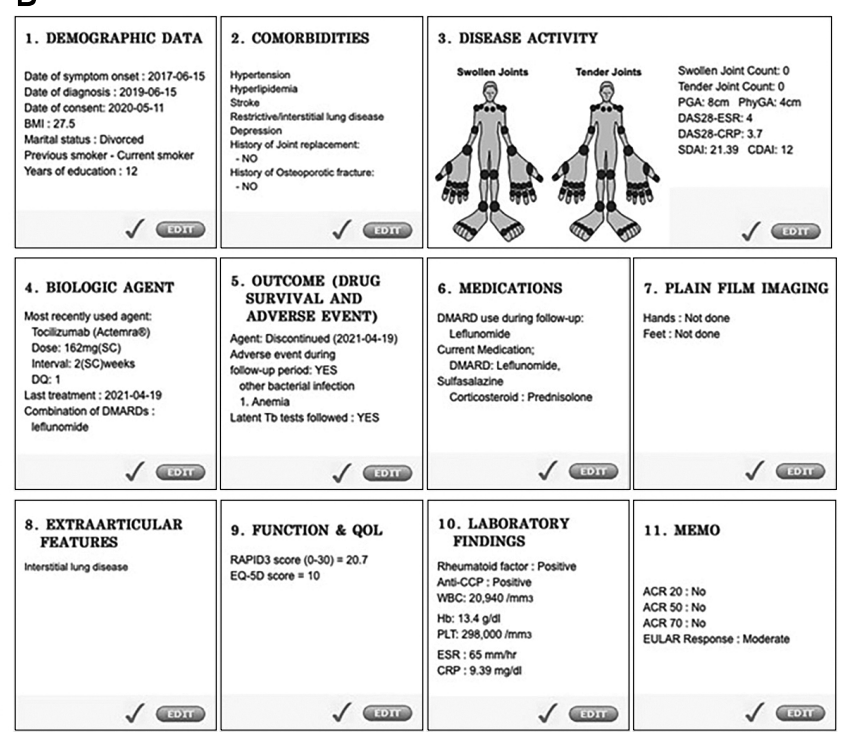

Figure 1. Dashboard images of initial (A) and follow-up (B) registration forms. 
roll an age- and gender-matched (1:1) non-biologic (conventional synthetic [cs-] DMARD) user on registration. However, soon after the control group was separated, it enrolled independently.

As a common format, the CRF of the three disease entities (RA, AS, PsA) identically consisted of 10 categories (Figure 1). Clinical parameters were customed to each disease within the respective category. The CRF was completed on follow-up. It obtained information including newly developed adverse events, switching or discontinuing an agent and its reasons, and others. After establishing the final version of the initial/follow-up CRFs, all elements were transformed into a web-based format that could be accessed anywhere, anytime. With the help of INFOrang Ltd. (Seoul, Korea), which codesigned the website and database system, KOBIO registered its first patient in December 2012. Since then, 47 institutes nationwide have participated in this quest. As of 31 December 2020, 2,471 patients with RA initiating or switching biologic therapy or tsDMARDs and 693 csDMARD-treated patients are registered in KOBIO along with over 2,000 patients with AS and close to 100 patients with PsA (Figure 2).

The electronic CRF format has enabled researchers to add or update various data sets seamlessly, including new agents, clinical parameters, newly reported adverse event of interest, and so on. A few new clinical parameters were added to CRF. The study team has kept adjustments to a minimum while readily updating new biologic agents, biosimilars, and Jak inhibitor as they are approved by the Korean Ministry of Food and Drug Safety. All adverse events in the KOBIO registry have been captured using the MedDRA (starting from version 17.0) since its launch. All registered terms have been verified by trained study nurses.

\section{MEDDRA WITHIN KOBIO}

Since mid-1990, ICH has developed MedDRA, a standardized medical terminology to facilitate sharing of regulatory information internationally for medical products. MedDRA is mainly used for registration, documentation, and safety monitoring by regulatory authorities and the biopharmaceutical industry during the regulatory process, ranging from clinical trials to post-marketing clinical research. After the release of version 1.0 in 1996, MedDRA has been updated twice a year. Its new version 24.0 was released in March 2021 [12]. Korean Ministry of Food and Drug Safety (MFDS) joined ICH in 2016. MedDRA can now be used to conduct clinical trial, approval, and adverse event report in Korea. Korean MFDS published guideline of MedDRA in 2019 [13].

MedDRA enables the classification of terms using a 5-level hierarchy. These terms are superordinate or subordinate to each other, from the broadest groups of terms to the more specific groups of term. Levels include System Organ Class (SOC), High-Level Group Terms (HLGT), High-Level Terms (HLT), Preferred Terms (PT), and Low-Level Terms (LLT) [14]. Depending on the level of specificity required, individual cases are coded with specificity using LLT terms (Figure 1).

MedDRA is used not only in clinical trials by regulatory authorities, but also in many cohorts and registers worldwide to classify comorbidities and adverse events [12]. Cohorts and registers are important to obtain evidence in effectiveness and safety of a medication and to guide clin-

Cumulative enrollment the KOBIO registry

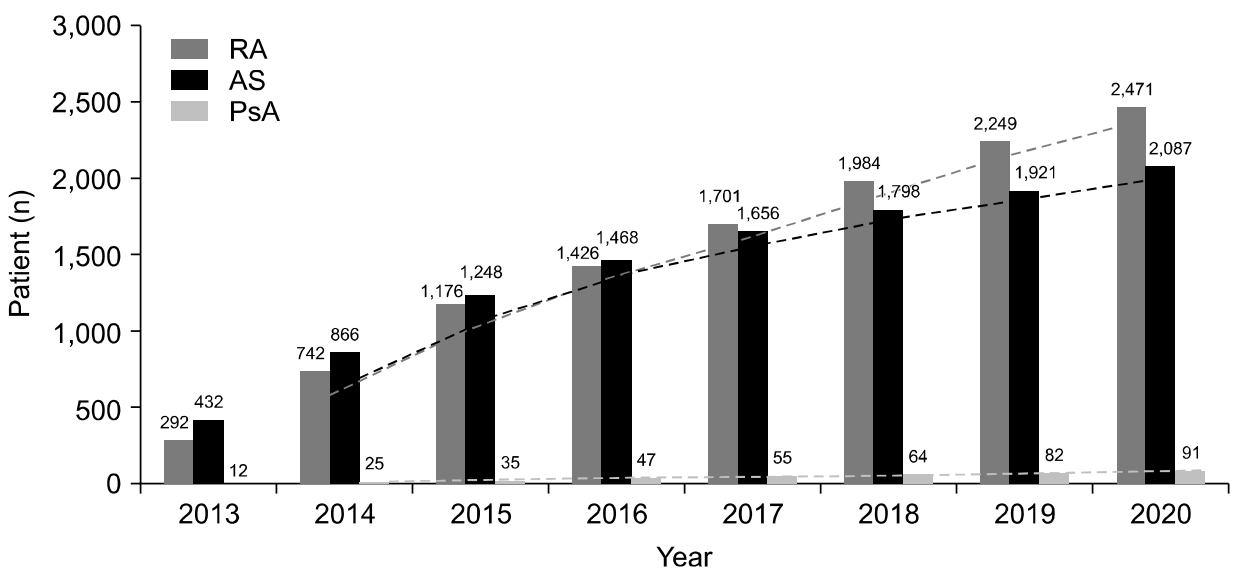

Figure 2. Cumulative enrollment in the $\mathrm{KOBIO}$ registry overtime on December 2020. KOBIO: Korean College of Rheumatology Biologics and targeted therapy, RA: rheumatoid arthritis, AS: ankylosing spondylitis, PsA: psoriatic arthritis. 
ical practice and health policy [15]. To improve gathering and sharing of data, a standardized terminology to assess adverse events and comorbidities is of the utmost importance. Thus, MedDRA is recommended to be used as the preferred dictionary for the coding of adverse events in European Biological Registries to ensure the homogeneity of data collection across registries [16]. Many registries in rheumatology such as the German (RABBIT), the British (BSRBR), the Portuguese (Reuma.pt), the Spanish (BIOBADASER), the Denmark (DANBIO), and the Norwegian (NOR-DMARD) use MedDRA system [17-22]. The KOBIO registry adopted MedDRA for reporting adverse events right after its launch and updated it to an electronic version of in 2019 (Figure 3), allowing researchers to easily select preferred terms on-line.

\section{FOOTSTEPS OF KOBIO}

Gathering full clinical, laboratory, and radiographic data of enrolled patients, especially during their follow-up, and data management are ongoing challenges of KOBIO. In addition, new treatment agents as well as biosimilars are being introduced every year. Minimizing follow-up loss is also important: registered patients often transfer to another rheumatology center. Last but not least, introducing the study format and electronic CRF of KOBIO to newcomers every year is sometimes demanding. To improve and maintain the quality of data obtained, the study team has conducted biannual workshops for participating physicians, nurses, and researchers since 2013. The program consists of lectures on registering web-based data, common queries, updates in CRFs and basic lectures on the treatment of RA, AS, and PsA. The workshop also provides an opportunity to directly interact and discuss with participants who engage in the task of entering data on-line. Because of the COVID-19 pandemic, an online workshop has been conducted since 2020 .

KOBIO has also been the database igniting important public health issues. For example, KCR presented results of the KOBIO data analysis of patients with RA on October 12, 2020, the World Arthritis Day [23]. Data from 2,379 patients with RA registered in the KOBIO from December 2012 to September 2020 were analyzed. The analysis showed that $52.6 \%$ of patients complained of severe pain (Visual Analogue Scale [VAS] $\geq 7$ points out of 10) owing to insufficient effects or side effects during treatment with csDMARDs. After using biologics or tsDMARDs, the proportion of patients who achieved DAS28 remission or low disease activity in the first year was $56.5 \%$. However, $21.5 \%$ of these patients still complained of uncomfortable pain (VAS $\geq 4$ points out of 10). Thus, irrespective of the increase of remission rate after the use of biologics and tsDMARDs, pain that affects the quality of life cannot be easily eliminated. The KCR concluded that research on new treatment strategies is needed to alleviate pain.

The KCR Research Committee has invited KCR members to analyze KOBIO data on various topics. Numerous abstracts have been presented at national and international conferences and published in peer-reviewed articles. A group of studies have investigated the association between comorbidities and disease activity or severity in patients treated with biologics [24-28]. One study has analyzed whether body mass index (BMI) is associated with disease activity markers and clinical manifestations in axial spondyloarthritis (SpA) [24]. Its results showed that increased BMI was closely associated with the presence of syndesmophytes, but not with disease activity indexes in axial SpA. A subsequent study has investigated effects of TNF inhibitors (TNFis) on renal

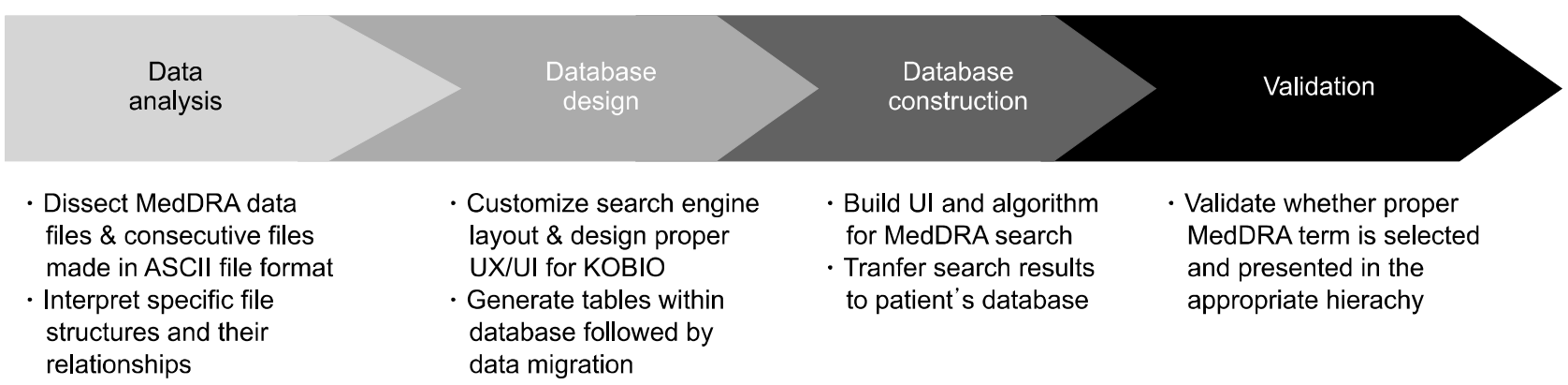

Figure 3. Schematic of developing the online version of MedDRA incorporated within KOBIO. MedDRA: Medical Dictionary for Regulatory Activities, KOBIO: Korean College of Rheumatology Biologics and targeted therapy, ASCII: American Standard Code for information interchange, UX: user experience, UI: user interface. 
function of patients with RA [26]. It found that TNFi did not alter renal function during treatment, and the authors concluded TNFi may be a safe treatment option irrespective of renal function of the patient.

Data from studying treatment responses to biologics or tsDMARDs "switchers" have been possible since the KOBIO registry monitors patients across different agents. One study investigated patterns of biologic therapy and reasons for switching biologics in Korean patients with RA [29]. The most common reason for switching biologics was inefficacy, followed by adverse events such as infusion reactions, infections, and skin eruptions. Recently, Min et al. [30] have compared the clinical efficacy and drug retention between TNFi-treated AS patients who switched to a different TNFi and those who switched to secukinumab. Both groups showed comparable clinical efficacy and drug retention in patients with previous exposure to a TNFi.

Adverse events or drug retention rates of biologics or tsDMARDs in elderly patients have been analyzed using the registry data [31-33]. Data on elderly patients with RA from clinical trials are limited owing to exclusion criteria based on age, comorbidities, or co-medication. A recent study has evaluated clinical outcomes and safety of biologics and tsDMARDs in elderly patients with RA $(n=355)$ and compared findings with those of patients treated with csDMARDs $(n=104)$ [33]. The estimated median retention of biologics/tsDMARDs was 2.5 years. Interstitial lung disease was the most common comorbidity associated with adverse events. In addition, several studies regarding efficacy, safety profiles, and drug retention of newly introduced biologics, tsDMARDs, and biosimilars in patients with RA and AS have been reported [34-36].

\section{FUTURE DIRECTIONS}

When KOBIO was launched in 2012, data entry began with seven biologic agents (infliximab, etanercept, adalimumab, tocilizumab, abatacept, rituximab, and golimumab). Currently, researchers can enter information of patients treated with an agent among 17 biologics or tsDMARDs. Since various treatment options and long-term follow-up are now possible, "switchers" not commonly found in randomized clinical trials could be further analyzed in the registry. These "switchers" can provide additional information regarding efficacy, safety, and others in addition to results from clinical trials [37]. Furthermore, individual reports of adverse events appear to improve pharmacovigilance on identifying rare and late-occurring events of special interest (ESI). KOBIO plans to continuously search for and investigate adverse events and ESI in Korean patients with rheumatic diseases that only could be pointed out via a long-term observation. Regarding big data analysis, KOBIO is planning to conduct a clinical research implementing precision medicine by merging or collaborating with other registries. To better illustrate emerging health policy issues, KOBIO will be able to assist the KCR by providing valuable RWD on drug survival, remission rates, functional aspects, and quality of life of patients. This would in turn will facilitate devising updates of treatment recommendations for Korean patients with rheumatic diseases.

\section{CONCLUSION}

Through the help and dedication of numerous staffs, researchers, stakeholders, and patients, KOBIO has become a nationwide web-based registry that can provide RWD to aid decision-making of clinicians to initiate, switch, or stop a biologic or tsDMARD. For researchers, KOBIO serves as a big data warehouse to help answer important research questions of various clinical aspects and drug safety issues. As an ongoing project, KOBIO will continue to monitor patients treated with earlier or newly approved agents of interest, ultimately offering information imperative for both clinicians and patients.

\section{ACKNOWLEDGMENTS}

We would like to thank Kimmie Hyrich, Kath Watson, Ms. Kim HJ and the KCR administrative staff, current and previous members of the study team, INFOrang, Ltd., and the generous donation of biopharmaceutical companies that made KOBIO possible.

\section{CONFLICT OF INTEREST}

No potential conflict of interest relevant to this article was reported.

\section{AUTHOR CONTRIBUTIONS}

Conceptualization: J.K., J.H.K., H.A.K., and K.S. Data acquisition: J.K., S.J.C., C.H.J., S-K.K., S.K.K., C.B.C., J.L., C.L., E.J.N., Y.B.P., S.S.L., T.H.K., S.H.P., J.Y.C., E.M.K., 
D.H.Y., Y.W.S., H.A.K., and K.S. Formal analysis: J.K., J.H.K., H.A.K., and K.S. Supervision: K.S. Writing: J.K., J.H.K., H.A.K., and K.S.

\section{REFERENCES}

1. Baumfeld Andre E, Reynolds R, Caubel P, Azoulay L, Dreyer NA. Trial designs using real-world data: the changing landscape of the regulatory approval process. Pharmacoepidemiol Drug Saf 2020;29:1201-12.

2. Sherman RE, Anderson SA, Dal Pan GJ, Gray GW, Gross T, Hunter NL, et al. Real-world evidence - what is it and what can it tell us? N Engl J Med 2016;375:2293-7.

3. Booth CM, Tannock IF. Randomised controlled trials and population-based observational research: partners in the evolution of medical evidence. Br J Cancer 2014;110:551-5.

4. Gliklich RE, Dreyer NA, Leavy MB. Patient registries. In: Gliklich RE, Dreyer NA, Leavy MB, eds. Registries for evaluating patient outcomes: a user's guide. 3rd ed. Rockville (MD), Agency for Healthcare Research and Quality (US), 2014.

5. Nikiphorou E, Buch MH, Hyrich KL. Biologics registers in RA: methodological aspects, current role and future applications. Nat Rev Rheumatol 2017;13:503-10.

6. Mercer LK, Regierer AC, Mariette X, Dixon WG, Baecklund E, Hellgren K, et al. Spectrum of lymphomas across different drug treatment groups in rheumatoid arthritis: a European registries collaborative project. Ann Rheum Dis 2017;76: 2025-30.

7. Mercer LK, Askling J, Raaschou P, Dixon WG, Dreyer L, Hetland ML, et al. Risk of invasive melanoma in patients with rheumatoid arthritis treated with biologics: results from a collaborative project of 11 European biologic registers. Ann Rheum Dis 2017;76:386-91.

8. Askling J, Fored CM, Brandt L, Baecklund E, Bertilsson L, Feltelius N, et al. Time-dependent increase in risk of hospitalisation with infection among Swedish RA patients treated with TNF antagonists. Ann Rheum Dis 2007;66: 1339-44.

9. Hetland ML, Christensen IJ, Tarp U, Dreyer L, Hansen A, Hansen IT, et al. Direct comparison of treatment responses, remission rates, and drug adherence in patients with rheumatoid arthritis treated with adalimumab, etanercept, or infliximab: results from eight years of surveillance of clinical practice in the nationwide Danish DANBIO registry. Arthritis Rheum 2010;62:22-32.

10. Iannone F, Gremese E, Atzeni F, Biasi D, Botsios C, Cipriani $\mathrm{P}$, et al. Longterm retention of tumor necrosis factor- $\alpha$ inhibitor therapy in a large Italian cohort of patients with rheumatoid arthritis from the GISEA registry: an appraisal of predictors. J Rheumatol 2012;39:1179-84.

11. Brown EG. Using MedDRA: implications for risk management. Drug Saf 2004;27:591-602.

12. MedDRA. Medical Dictionary for Regulatory Activities [Internet]. McLean (VA): MedDRA [cited 2021 Mar]. Available from: http://www.meddra.org/.

13. Ministry of Food and Drug Safety. ICH distributes the Korean version of International Medical Terminology (MedDRA) [Internet]. Cheongju: Ministry of Food and
Drug Safety, 2019 Sep 11 [cited 2021 Jun 4]. Available from: https://www.mfds.go.kr/brd/m_99/down.do?brd_id= ntc0021\&seq $=43693 \&$ data_tp $=$ A $\&$ file_seq $=1$.

14. MedDRA. Introductory Guide MedDRA version 23.0 [Internet]. McLean (VA): MedDRA, 2020 Mar [cited 2021 Jun 5]. Available from: https://admin.new.meddra.org/sites/default/files/guidance/file/intguide_\%2023_0_English.pdf.

15. Zavada J, Dixon WG, Askling J. Launch of a checklist for reporting longitudinal observational drug studies in rheumatology: a EULAR extension of STROBE guidelines based on experience from biologics registries. Ann Rheum Dis 2014;73:628.

16. Zink A, Askling J, Dixon WG, Klareskog L, Silman AJ, Symmons DP. European biologicals registers: methodology, selected results and perspectives. Ann Rheum Dis 2009;68:1240-6.

17. Canhão H, Faustino A, Martins F, Fonseca JE. Reuma.pt the rheumatic diseases Portuguese register. Acta Reumatol Port 2011;36:45-56.

18. Carmona L, de la Vega M, Ranza R, Casado G, Titton DC, Descalzo MÁ, et al. BIOBADASER, BIOBADAMERICA, and BIOBADADERM: safety registers sharing commonalities across diseases and countries. Clin Exp Rheumatol 2014;32(5 Suppl 85):S-163-7.

19. Kvien TK, Heiberg, Lie E, Kaufmann C, Mikkelsen K, Nordvåg BY, et al. A Norwegian DMARD register: prescriptions of DMARDs and biological agents to patients with inflammatory rheumatic diseases. Clin Exp Rheumatol 2005;23(5 Suppl 39):S188-94.

20. Silman A, Symmons D, Scott DG, Griffiths I. British Society for Rheumatology Biologics Register. Ann Rheum Dis 2003;62(Suppl 2):ii28-9.

21. Zink A, Listing J, Kary S, Ramlau P, Stoyanova-Scholz M, Babinsky K, et al. Treatment continuation in patients receiving biological agents or conventional DMARD therapy. Ann Rheum Dis 2005;64:1274-9.

22. Hetland ML, Unkerskov J, Ravn T, Friis M, Tarp U, Andersen LS, et al. Routine database registration of biological therapy increases the reporting of adverse events twentyfold in clinical practice. First results from the Danish Database (DANBIO). Scand J Rheumatol 2005;34:40-4.

23. Lee SY. KOBIO data analysis for World Arthritis Day [Internet]. Seoul: The Medical Herald, 2020 Oct 13 [cited 2021 Jul 9]. Available from: http://www.mediherald.com/ news/articleView.html?idxno $=61666$.

24. Kim SK, Choe JY, Lee SS, Shin K. Body mass index is related with the presence of syndesmophyte in axial spondyloarthritis: data from the Korean College of Rheumatology BIOlogics (KOBIO) registry. Mod Rheumatol 2017;27: 855-61.

25. Kim SK, Kwak SG, Choe JY. Association between biologic disease modifying anti-rheumatic drugs and incident hypertension in patients with rheumatoid arthritis: results from prospective nationwide KOBIO Registry. Medicine (Baltimore) 2020;99:e19415.

26. Kim SK, Choe JY, Kwak SG, Bae J, Park SH, Lee H. Effect of tumour necrosis factor-alpha inhibitors on renal function in patients with rheumatoid arthritis from the KOBIO registry from 2012 to 2016. Clin Exp Rheumatol 2018;36:1022-30.

27. Min HK, Kim HR, Lee SH, Shin K, Kim HA, Park SH, et al. Four-year follow-up of atherogenicity in rheumatoid arthri- 
tis patients: from the nationwide Korean College of Rheumatology Biologics Registry. Clin Rheumatol 2021; 40:3105-13.

28. Kim Y, Park S, Kim HS. The effect of extra-articular manifestations on tumor necrosis factor- $\alpha$ inhibitor treatment duration in patients with ankylosing spondylitis: nationwide data from the Korean College of Rheumatology BIOlogics (KOBIO) registry. Clin Rheumatol 2018;37:3275-84.

29. Park DJ, Choi SJ, Shin K, Kim HA, Park YB, Kang SW, et al. Switching profiles in a population-based cohort of rheumatoid arthritis receiving biologic therapy: results from the KOBIO registry. Clin Rheumatol 2017;36:1013-22.

30. Ki Min H, Kim HR, Lee SH, Hong YS, Kim MY, Park SH, et al. Retention rate and effectiveness of secukinumab vs TNF inhibitor in ankylosing spondylitis patients with prior TNF inhibitor exposure. Rheumatology (Oxford) 2021 Mar 16 [Epub]. DOI:10.1093/rheumatology/keab245.

31. Jung SM, Lee SW, Song JJ, Park SH, Park YB. Drug survival of biologic therapy in elderly patients with rheumatoid arthritis compared with nonelderly patients: results from the Korean College of Rheumatology Biologics registry. J Clin Rheumatol 2020 Dec 14 [Epub]. DOI:10.1097/RHU.0000 000000001644.

32. Jung SM, Kwok SK, Ju JH, Lee SW, Song JJ, Yoon CH, et al. Risk factors associated with inadequate control of disease activity in elderly patients with rheumatoid arthritis: results from a nationwide KOrean College of Rheumatology BIOlogics (KOBIO) registry. PLoS One 2018;13:e0205651.

33. Koh JH, Lee SK, Kim J, Kim HA, Shin K, Min JK. Effectiveness and safety of biologic and targeted synthetic disease-modifying anti-rheumatic drugs in elderly patients with rheumatoid arthritis: real-world data from the KOBIO Registry. Clin Exp Rheumatol 2021;39:269-78.

34. Kim HA, Lee E, Lee SK, Park YB, Lee YN, Kang HJ, et al. Retention rate and safety of biosimilar CT-P13 in rheumatoid arthritis: data from the Korean College of Rheumatology Biologics registry. BioDrugs 2020;34:89-98.

35. Kim HA, Lee E, Lee SK, Park YB, Lee YN, Kang HJ, et al. Retention rate and long-term safety of biosimilar CT-P13 in patients with ankylosing spondylitis: data from the Korean College of Rheumatology Biologics registry. Clin Exp Rheumatol 2020;38:267-74.

36. Kim HA, Lee E, Lee SK, Park YB, Shin K. Retention rate and efficacy of the biosimilar CT-P13 versus reference infliximab in patients with ankylosing spondylitis: a propensity score-matched analysis from the Korean College of Rheumatology Biologics Registry. BioDrugs 2020;34:529-39.

37. Hetland ML. DANBIO: a nationwide registry of biological therapies in Denmark. Clin Exp Rheumatol 2005;23(5 Suppl 39):S205-7. 